\title{
GLOBAL PRICING POLICIES AND COST-EFFECTIVENESS OF TRASTUZUMAB IN LATIN AMERICA
}

\section{Robert B. Giffin \\ Medtronic, Minneapolis, MN \\ robert.b.giffin@medtronic.com}

Pinchon-Riviere and colleagues have made an extremely timely contribution that addresses the thorny issues of innovative, high-cost medicines, global health disparities, and the growing interest and application of HTA in low- and middle-income countries.

Innovative, high-cost drugs can dramatically improve the treatment options for patients, but can also create serious financial challenges for health systems. While the United States and other high-income countries are confronting the new reality of medicines that can cost $\$ 50,000$ or more per patient, access to high-value, high-cost medicines nevertheless remains high. In low- and middle-income countries, however, high-cost medicines are far more likely to be out of reach to the majority of the population. As Pinchon and his colleagues point out, there is little rationality in global drug pricing - prices for many drugs are actually higher in low- and middle-income countries than in high-income countries. Proceeding from this surprising revelation, the authors have developed a valuable cost-effectiveness framework for understanding global pharmaceutical pricing.

The authors examine the pricing of trastuzumab, a targeted therapy that has been controversial for its high cost, even while it has become the standard of care for adjuvant therapy in the United States and other high-income countries for HER2positive breast cancer patients. The authors' model the costeffectiveness of treatment with and without trastuzumab for seven Latin American countries at current prices and based on local epidemiology. They further estimate the prices that would make trastuzumab cost-effective in each. Their results show that incremental cost effectiveness ratios for trastuzumab are similar to those in high-income countries. However, when viewed from the perspective of national wealth, the authors found wide disparities between low- and middle-income and high-income countries. The Latin American countries they studied would have to spend between 3.6 and 35.5 times their per capita gross domestic product to achieve just one quality-adjusted life-year (QALY) using trastuzumab. (The per capita gross domestic product [GDP] per QALY is a commonly used standard, and indeed in high-income countries, incremental cost-effectiveness ratios [ICERs] for trastuzumab have been found to be close to one per capita GDP per QALY.) In terms of current healthcare spending, the results are even starker - an additional QALY would require between 60 and 826 times current levels of per capita health spending. The bottom line is that many patients in these countries will not have access to this therapy unless there are dramatic changes in the way trastuzumab is priced.

The authors do not explore the many factors that have led to current global pharmaceutical pricing approaches, nor do they wade into detailed policy prescriptions, other than to suggest the adoption of some sort of differential pricing. Rather, their main contribution is to demonstrate a viable and rigorous analytic framework that can inform policy. This is badly needed, as future drugs are now in the pipeline that will pose new challenges to financial health systems. And as health technology assessment becomes increasingly established in low- and middle-income countries as a tool for allocating healthcare resources, there is a risk that access to new medicines that do not meet some sort of objective pricing threshold will be severely constrained. The detailed methodology described by the authors provides a readily replicable approach that can be applied to new medicines in any country, and it thus provides a useful backdrop for policymakers attempting to address pricing issues. Having a commonly understood framework for assessing global pricing expectations and realities will give all stakeholders better opportunities for establishing global pricing approaches that maximize access to critical new medicines. 